\title{
Nonlinear water waves in channels of arbitrary shape
}

\author{
By MICHELLE H. TENG AND THEODORE Y. WU \\ Engineering Science, 104-44, California Institute of Technology, Pasadena, CA 91125, USA
}

(Received 9 August 1991 and in revised form 3 February 1992)

The generalized channel Boussinesq $(\mathrm{gcB})$ two-equation model and the forced channel Korteweg-de Vries (cKdV) one-equation model previously derived by the authors are further analysed and discussed in the present study. The gcB model describes the propagation and generation of weakly nonlinear, weakly dispersive and weakly forced long water waves in channels of arbitrary shape that may vary both in space and time, and the $\mathrm{cKdV}$ model is applicable to unidirectional motions of such waves, which may be sustained under forcing at resonance of the system. These two models are long-wave approximations of a hierarchy set of section-mean conservation equations of mass, momentum and energy, which are exact for inviscid fluids. Results of these models are demonstrated with four specific channel shapes, namely variable rectangular, triangular, parabolic and semicircular sections, in which case solutions are obtained in closed form. In particular, for uniform channels of equal mean water depth, different cross-sectional shapes have a leading-order effect only on the variations of a $\kappa$-factor of the coefficient of the term bearing the dispersive effects in the model equations. For this case, the uniform-channel analogy theorem enunciated here shows that long waves of equal (mean) height in different uniform channels of equal mean depth but distinct $\kappa$-shape factors will propagate with equal velocity and with their effective wavelengths appearing $\kappa$ times of that in the rectangular channel, for which $\kappa=1$. It also shows that the further channel shape departs from the rectangular, the greater the value of $\kappa$. Based on this observation, the solitary and cnoidal waves in a $\kappa$-shaped channel are compared with experiments on wave profiles and wave velocities. Finally, some three-dimensional features of these solitary waves are presented for a triangular channel.

\section{Introduction}

This paper is a further extension of the previous studies by Teng (1990) and Teng \& Wu (1990), in which two theoretical models, namely the generalized channel Boussinesq ( $\mathrm{gcB}$ ) model for bidirectional motions and the forced channel Kortewegde Vries (cKdV) model for unidirectional motions were developed to describe weakly nonlinear and weakly dispersive long water waves generated and propagating in a quasi-one-dimensional variable channel. These models are more general than the previous versions in allowing channel boundary variations both in space and time, and in admitting moving boundaries as forcing disturbances that can be sustained at resonance of the system for an extended period of time.

The general subject of nonlinear long waves evolving in a non-uniform medium has been a field of considerable activity and development. For uniform channels, Peters (1966), Peregrine (1968), Shen (1968, 1969) and Fenton (1973) investigated waves travelling in a straight uniform channel with arbitrary cross-section. In studies of the 
nonlinear evolution of long waves on water of variable depth, a set of evolution equations of the Boussinesq class was derived by Peregrine (1967) and by Madsen \& Mei (1969) independently. The transient procedure of fission of an initial solitary wave into a sequence of solitary waves after climbing up a shelf was given in numerical results by Madsen \& Mei and was supported by Johnson (1973). For rectangular channels with variable depth and width, Shuto (1974) obtained a KdVtype equation with variable coefficients as a theoretical model. Similar model equations were derived by Shen \& Zhong (1981) and Cai \& Shen (1985) for variable triangular and elliptical channels. In analysing the wave conservation properties, Miles $(1979,1980)$ pointed out that Shuto's model does not conserve mass owing to neglect of the reflected waves but it is adiabatically invariant in energy. For this category of channel shape, Chang, Melville \& Miles (1979) carried out numerical and experimental studies on solitary waves travelling in a rectangular channel with a linearly varying width but a uniform depth, using Shuto's equation for numerical computation. For studying the run-up of long waves on sloping beaches, the Lagrangian description was used by Pederson \& Gjevik (1983) to develop a Boussinesq-class model. Also in terms of the Lagrangian description, Zelt (1986) and Zelt \& Raichlen (1990) derived a set of long-wave equations to study wave responses to bays and harbours with variable bathymetry and sidewall shape for some simple configurations. For wide channels of arbitrary shape, David, Levie \& Winternitz (1987) introduced a generalized Kadomtsev-Petviashvili ( $\mathrm{K}-\mathrm{P}$ ) equation (with variable coefficients) as a weakly three-dimensional long-wave model for simulating solitary waves in a wide and shallow marine strait. The forced KadomtsevPetviashvili equation has also been analysed and applied by Mathew \& Akylas (1990) to study the propagation of weakly nonlinear long water waves along a wide (compared with the depth) channel bounded by sloping sidewalls. (Further references to the literature may be found in Teng \& Wu 1990.) In these studies, channel boundaries have varied only in space but not with time. Also, external forcings have not been considered.

The main objectives of our series of studies are (i) to present a set of integral conservation laws for a segment of free-surface flow along a channel of arbitrary variable shape; (ii) to provide a recapitulation of the two theoretical models adopted here for further applications; (iii) to determine the general features of solitary and cnoidal waves propagating along a uniform channel of arbitrary shape; (iv) to evaluate the evolution of nonlinear long waves in converging-diverging channels of variable depth and width; and $(v)$ to explore the generation of waves by forcing excitations sustained at resonance of the system. The first three parts will be addressed in the present article.

The general case under consideration is a class of channels whose width and depth are of the same order in dimension, the water depth is small compared with a typical length of the prevailing waves, and the channel boundaries may vary gradually along the channel and possibly with time to act as an external forcing disturbance. For this general case, a set of section-mean equations of motion is presented in $\$ 2$, which are exact for inviscid fluids. From these basic equations a number of integral conservation laws are derived for a segment of wave motions bounded by any two channel sections. These fundamental conservation properties of nonlinear channel waves are valuable for assessing the validity of theoretical models and numerical results as well as providing a sound basis for deriving the desired long-wave models. In $§ 3$, a summary is given of the derivation of the two theoretical models, the gcB for bidirectional long waves and the cKdV for unidirectional long waves being 


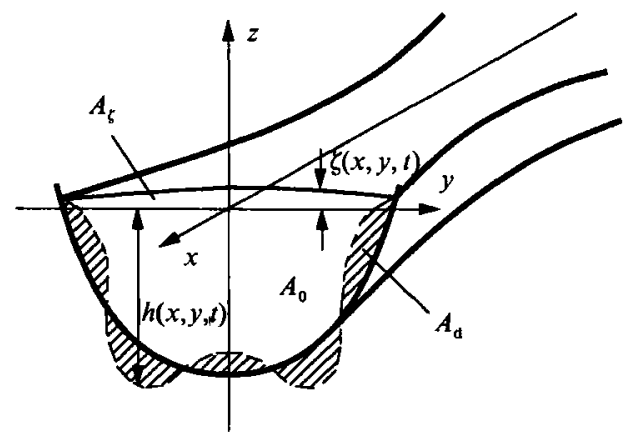

Figure 1. A channel with variable cross-section of area $A(x, t)=A_{0}(x)-A_{\mathrm{d}}(x, t)+A_{\xi}(x, t), A_{0}$ being the unperturbed fixed sectional wetted area, $A_{\mathrm{d}}$ the wetted area variation due to a moving boundary of the channel and $A_{\zeta}$ the wetted area variation due to free-surface motion.

produced and propagating in a variable channel of arbitrary shape. In $\$ 4$, the effects of channel configuration are examined for four specific shapes, i.e. variable rectangular, triangular, parabolic, and semicircular cross-sections, for which the solutions are obtained in closed form. The results show that variations in channel shape only affect the value of a parametric factor, called the shape factor $\kappa$, of the coefficient of the term bearing the dispersive effects in the model equations for channels of equal mean depth. For uniform channels in particular, an arbitrary channel characterized by a specific shape factor $\kappa$ can be uniquely correlated, by a proper scaling transformation only involving $\kappa$, with an analogous rectangular channel. On this basis we proposed a uniform-channel analogy theorem, by which the wave properties in a $\kappa$-shaped uniform channel are reduced to a calculation of the waves in the analogous rectangular channel, a problem which is classical. This theorem holds not only for free waves, but also for waves produced by forcing disturbances. By applying this theorem, the existence of solitary and cnoidal waves travelling in permanent form along a uniform channel of arbitrary shape are analysed and discussed in $\$ 5$ for both the $\mathrm{gcB}$ and $\mathrm{cKdV}$ models. Comparison between theory and experiment on wave profiles and wave velocities are made for some representative cases. Finally, three-dimensional features of the wave profile are recovered for a solitary wave propagating along a triangular channel.

\section{Channel section-mean equations}

We recapitulate here, as a basis for further applications and discussions, the basic theoretical models developed by Teng (1990) and Teng \& Wu (1990) for propagation and generation of long waves in variable channels of arbitrary shape. A typical water channel of the general group under consideration is shown in figure 1. The channel is straight, symmetric about its $(x, z)$-centreplane, with cross-sections arbitrary in shape and with its area gradually varying along the channel and possibly changing slowly in time. The width and depth of the channel are supposed to be of the same order, and there are no excessively sloped banks. The water in the channel is supported from underneath by the bottom at $z=-h(x, y, t)$, with its free surface displaced at time $t$ to $z=\zeta(x, y, t)$, and with the free surface extended across the channel to the waterline at $y= \pm b(x, t)$, at which the bank may be vertical or inclined.

We consider the class of motion of three-dimensional water waves of arbitrary 
amplitude and wavenumber, propagating in two horizontal dimensions along a channel as prescribed. The water in the channel will be assumed inviscid and incompressible, with constant density $\rho$. The effects of surface tension will be neglected.

The motion of the water, with velocity $U=(u, v, w)$ and the pressure $p(x, y, z, t)$, satisfies the Euler equations

$$
\begin{gathered}
\boldsymbol{\nabla} \cdot \boldsymbol{U}=0, \\
\frac{\mathrm{d} \boldsymbol{U}}{\mathrm{d} t} \equiv U_{t}+\boldsymbol{U} \cdot \boldsymbol{\nabla} \boldsymbol{U}=-\frac{1}{\rho} \boldsymbol{\nabla} p_{\mathrm{e}} \quad\left(p_{\mathrm{e}}=p+\rho g z\right),
\end{gathered}
$$

and the boundary conditions

$$
\begin{gathered}
w=\frac{\mathrm{d} \zeta}{\mathrm{d} t}=\zeta_{t}+u \zeta_{x}+v \zeta_{y}, \quad z=\zeta(x, y, t), \quad|y|<b(x, t) \\
p=p_{\mathrm{a}}(x, y, t), \quad z=\zeta(x, y, t), \quad|y|<b(x, t) \\
w=-\frac{\mathrm{d} h}{\mathrm{~d} t}=-\left(h_{t}+u h_{x}+v h_{y}\right), \quad z=-h(x, y, t) \\
v= \pm\left(\hat{b}_{t}+u \hat{b}_{x}\right), \quad y= \pm \hat{b}(x, t)
\end{gathered}
$$

where $\boldsymbol{\nabla}=\left(\partial_{x}, \partial_{y}, \partial_{z}\right)$, the variable subscripts denoting differentiation, $p_{\mathbf{e}}$ is the excess pressure, $p_{\mathrm{a}}$ the specified ambient surface pressure, and $g$ the constant acceleration due to gravity. Condition (6) applies to any vertical segment (of halfwidth $\hat{b}$ ) of the sidewalls. The free-surface flow will be assumed irrotational, with $\boldsymbol{U}=\boldsymbol{\nabla} \phi$, where the velocity potential $\phi(x, y, z, t)$ satisfies the Laplace equation,

$$
\nabla^{2} \phi=0
$$

and we also have the Bernoulli equation

$$
\frac{\partial \phi}{\partial t}+\frac{1}{2} U^{2}+\frac{p}{\rho}+g z=\frac{p_{\infty}}{\rho} .
$$

For our general purpose, it is of basic interest to first derive a set of onedimensional section-mean equations by integrating (1) and (2) over the wetted crosssectional area of the channel flow, so that the dominant mean wave motion in the $x$ direction can be precisely prescribed. This set of section-mean equations is exact for an inviscid and incompressible fluid, without having the motion limited to small amplitudes, or to waves being long, or even to flows being irrotational.

First, we define the section-mean value of any flow quantity $f(x, y, z, t)$ as

$$
\bar{f}(x, t) \equiv \frac{1}{A} \iint_{A} f(x, y, z, t) \mathrm{d} y \mathrm{~d} z \equiv \frac{1}{A}\langle f\rangle,
$$

where $A$ is the wetted cross-sectional area, which, like the free surface, is as yet unknown. By applying Reynolds' transport theorem to the flow in a material volume $V(t)$ which at time $t$ coincides with a vertical column of water lying between any two sections of specified $x$, it is readily shown (Teng 1990), using the kinematic conditions $(3),(5)$ and (6), that for a smooth flow variable $f(x, y, z, t)$,

$$
\left\langle\frac{\mathrm{d} f}{\mathrm{~d} t}\right\rangle=\frac{\partial}{\partial t}\langle f\rangle+\frac{\partial}{\partial x}\langle u f\rangle,
$$




$$
A \frac{\overline{\mathrm{d} f}}{\mathrm{~d} t}=\frac{\partial}{\partial t}(A \bar{f})+\frac{\partial}{\partial x}(A \overline{u f})
$$

where $u$ is the $x$-component of $U=(u, v, w)$.

By applying this channel-transport theorem to (1), (2) and (8), taking in turn $f=1, u, v, w$ and

$$
f=H \equiv \frac{1}{2} \rho\left(u^{2}+v^{2}+w^{2}\right)+\rho g z
$$

we obtain the following section-mean equations,

$$
\begin{gathered}
A_{t}+(A \bar{u})_{x}=0 \\
(A \bar{u})_{t}+(A \overline{u u})_{x}=-\frac{A}{\rho} \overline{p_{x}} \\
(A \bar{v})_{t}+(A \overline{u v})_{x}=-\frac{A}{\rho} \overline{p_{\mathrm{e} y}}=\frac{1}{\rho} \int_{-b}^{b}\left[\left(p_{\mathrm{e}}\right)_{\zeta} \frac{\partial \zeta}{\partial y}+\left(p_{\mathrm{e}}\right)_{-h} \frac{\partial h}{\partial y}\right] \mathrm{d} y \\
(A \bar{w})_{t}+(A \overline{u w})_{x}=-\frac{A}{\rho} \overline{p_{\mathrm{e} z}}=\frac{1}{\rho} \int_{-b}^{b}\left[\left(p_{\mathrm{e}}\right)_{-h}-\left(p_{\mathrm{e}}\right)_{\zeta}\right] \mathrm{d} y \\
(A \bar{H})_{t}+[A(\overline{H u}+\overline{p u})]_{x}=A \bar{p}_{t}-\frac{\partial}{\partial t}(A \bar{p})
\end{gathered}
$$

where $(\cdot)_{\zeta}$ and $(\cdot)_{-h}$ denote the quantity evaluated at $z=\zeta$ and $z=-h$, respectively. These five equations give the section-mean conservation laws for mass, horizontal and vertical momenta and energy, noting that $\langle H\rangle$ is the sum of the kinetic and potential energy density of a water slab of unit thickness in $x$. These basic equations are exact for inviscid fluids, whether or not the flow is irrotational. However, this hierarchy set of equations is not closed, there being more unknowns than the number of equations. Nevertheless, they provide a set of exact local conservation laws that should be of fundamental interest, for example, for assessing the accuracy of approximate and numerical solutions.

A set of integral conservation laws can be derived under the assumptions that (i) the channel spans over $x \in \Re$; (ii) all the resulting integrals are convergent; and (iii) $h(x, y, t)=h_{0}(x, y)-h_{\mathrm{d}}(x, y, t)$, of which the moving disturbance part, $h_{\mathrm{d}}$, is assumed integrable over the water planform $S_{\infty}(-\infty<x<\infty,|y|<b(x, t))$. Thus upon integrating (1) over $S\left(x_{1}<x<x_{2},|y|<b(x, t)\right)$, which is an arbitrary segment of $S_{\infty}$, we have for the excess mass $m$ within $S$ the conservation law

$$
\frac{\mathrm{d} m}{\mathrm{~d} t}=\dot{m} \equiv \rho \int_{x_{1}}^{x_{2}} A_{t} \mathrm{~d} x=Q_{1}-Q_{2},
$$

where $Q_{j}=Q\left(x_{j}\right)(j=1,2), Q(x)=\rho A \bar{u}$ being the discharge across the section at $x$. For the excess mass centre within the segment $S, x_{c}(t)$ say, defined as

$$
m x_{\mathrm{c}}=\rho \int_{x_{1}}^{x_{2}} A x \mathrm{~d} x
$$

we obtain the impulse equation

$$
\frac{\mathrm{d}}{\mathrm{d} t}\left(m x_{\mathrm{c}}\right)=x_{1} Q_{1}-x_{2} Q_{2}+I_{1} \quad\left(I_{1}=\int \rho A \bar{u} \mathrm{~d} x\right),
$$


$I_{1}$ being the $x$-component of impulse of the fluid within the volume segment of $S$. Further, for the balance of the $x$-component of the momentum we have

$$
\dot{I}_{1}=\int \mathrm{d} S \int_{-h}^{\zeta} \rho \frac{\mathrm{d} u}{\mathrm{~d} t} \mathrm{~d} z=-\int \mathrm{d} S \int_{-h}^{\zeta} \frac{\partial}{\partial x}\left(\rho u^{2}+p_{\mathrm{e}}\right) \mathrm{d} z,
$$

which yields for flows that are symmetric about $y=0$ the expression

$$
\dot{I}_{1}=\left(\Pi_{11}\right)_{1}-\left(\Pi_{11}\right)_{2}+2 \int \frac{\partial b}{\partial x} \mathrm{~d} x \int_{-h}^{\zeta}\left(p_{\mathrm{e}}\right)_{b} \mathrm{~d} z+\int \mathrm{d} x \int_{-b}^{b}\left[\left(p_{\mathrm{e}}\right)_{5} \frac{\partial \zeta}{\partial x}+\left(p_{\mathrm{e}}\right)_{-h} \frac{\partial h}{\partial x}\right] \mathrm{d} y,
$$

where $\Pi_{11}=A\left(\rho \overline{u u}+\overline{p_{\mathrm{e}}}\right)$, the $x$-component of the total sectional momentum flux, and the first integral is the contribution from vertical sidewalls, if any, at which $\left(p_{\mathrm{e}}\right)_{b}=p_{\mathrm{e}}(x, b, z, t)$.

For the vertical component of momentum we have

$$
\dot{I}_{3}=\frac{\mathrm{d}}{\mathrm{d} t} \int \rho w \mathrm{~d} V=\left(\Pi_{13}\right)_{1}-\left(\Pi_{13}\right)_{2}+\int_{S}\left[\left(p_{\mathrm{e}}\right)_{-h}-\left(p_{\mathrm{e}}\right)_{\zeta}\right] \mathrm{d} S,
$$

where $\Pi_{13}=\rho A \bar{u}$. Finally, for the total energy we have from (16) the relation

$$
\dot{E}=\mathscr{F}_{1}-\mathscr{F}_{2}-2 \int \mathrm{d} S \int_{-h}^{\zeta} b_{t}(p)_{b} \mathrm{~d} z-\int_{S}\left(\zeta_{t} p_{\zeta}+h_{t} p_{-h}\right) \mathrm{d} S,
$$

where $\mathscr{F}=A \overline{(H+p) u}$, the total sectional energy flux. Here, the first integral may prevail should the channel sidewalls be vertical and moving transversely.

If the flow disturbance has a compact support, i.e. all the physical quantities perturbed from the state of rest are assumed to be regular at $x= \pm \infty$ such that all the integrals involving them are convergent, then by letting $x_{1} \rightarrow-\infty, x_{2} \rightarrow+\infty$, we find that all the above relations hold provided that all of

$$
Q_{j}, \quad x_{j} Q_{j}, \quad\left(\Pi_{11}\right)_{j}, \quad\left(\Pi_{13}\right)_{j}, \quad \mathscr{F}_{j} \rightarrow 0 \quad(j=1,2) .
$$

These integral conservation laws are exact for inviscid fluids. Their physical significance is quite evident as the relations appear almost in the classical form of mechanics. For instance, by (19), the fluid impulse $I_{1}$ increases over the transport by the total momentum flux at a rate equal to the longitudinal component of the surface pressure acting on the flow boundary, as expected. These relationships are general in that they are not limited to motions being small or irrotational, or waves being long, or to particular boundary configurations. For some special cases they agree with those previously discussed by Wu $(1979,1981)$, Benjamin \& Mahony (1971) and by Keulegen \& Patterson (1940).

\section{Channel long-wave models}

For channel long waves, we note that the first two equations of the set (12)-(16) are sufficient for determining the two mean-flow variables since the closure can be effected by expansion, for long waves, of the field equation (7) for the velocity potential $\phi$ and the Bernoulli equation (8). We outline below the derivation of the two theoretical models given by Teng (1990), Teng \& Wu (1990) for modelling long waves in variable channels, with major steps delineated to make this paper selfcontained. 
The total wetted cross-sectional area in general consists of three parts:

$$
A(x, t)=A_{0}(x)-A_{\mathrm{d}}(x, t)+A_{\zeta}(x, t) \equiv A_{h}(x, t)+A_{\zeta}(x, t),
$$

where $A_{0}$ is the unperturbed cross-sectional wetted area, $A_{\mathrm{d}}$ the wetted area variation due to a moving boundary of the channel or a moving submerged body as a forcing function, and $A_{\zeta}$ is the wetted area variation due to wave motion at the free surface, which can be written $A_{\zeta}=2 b \tilde{\zeta}$, where

$$
\widetilde{(\cdot)}(x, t)=\frac{1}{2 b} \int_{-b}^{b}(\cdot)(x, y, z=\zeta(x, y, t), t) \mathrm{d} y
$$

is the 'sectional free-surface mean' of $(\cdot)$. Thus (12) can be written as

$$
(2 b \tilde{\zeta})_{t}+\left[\left(A_{h}+2 b \tilde{\zeta}\right) \bar{u}\right]_{x}=-\left(A_{h}\right)_{t} .
$$

This section-mean continuity equation, which is exact, gives a relation between $\tilde{\zeta}$ and $\bar{u}$ in terms of $b$ and $A_{h}$ as functions of $x$ and $t$. The closure of the system is accomplished by relating the unknown variables $\overline{u u}$ and $\bar{p}_{x}$ in (13) to the original two unknowns $\tilde{\zeta}$ and $\bar{u}$. This is achieved, to a second-order approximation, by applying the following perturbation expansions appropriate for long waves to the Laplace equation (7) and the Bernoulli equation (8).

\subsection{The generalized channel Boussinesq model}

With the vertical lengths scaled by a typical constant mean water depth $h_{\mathrm{c}}$, the horizontal lengths by a typical wavelength $\lambda$, time by $\lambda / c_{0}$, where $c_{0}=\left(g h_{\mathrm{c}}\right)^{\frac{1}{2}}$ is the linear critical wave speed, the flow is found to be characterized by two important parameters,

$$
\alpha=a / h_{\mathrm{c}}, \quad \epsilon=h_{\mathrm{c}} / \lambda,
$$

where $a$ is a typical wave amplitude. The theoretical models that were derived by Teng (1990) and Teng \& Wu (1990) are of the Boussinesq class of wave motion specified by $\alpha=O\left(\epsilon^{2}\right) \ll 1$, so that the nonlinear effects (scaled by $\alpha$ ) and the dispersive effects (scaled by $\epsilon^{2}$ ) are both well matched with the net inertial effects.

In dimensionless form, (7) and (8) become

$$
\begin{gathered}
\phi_{x x}+\frac{1}{\epsilon^{2}}\left(\phi_{y y}+\phi_{z z}\right)=0, \\
p+\phi_{t}+\frac{1}{2}\left[\phi_{x}^{2}+\frac{1}{\epsilon^{2}}\left(\phi_{y}^{2}+\phi_{z}^{2}\right)\right]+z=0,
\end{gathered}
$$

and the boundary conditions in the cross-flow plane become

$$
\begin{gathered}
\left.\frac{\partial \phi}{\partial n}\right|_{z-\zeta}=\epsilon^{2} \frac{\left(\zeta_{t}+u \zeta_{x}\right)}{\left(1+\zeta_{y}^{2}\right)^{\frac{1}{2}}}, \\
\left.p\right|_{z-\zeta}=p_{\mathrm{a}}, \\
\left.\frac{\partial \phi}{\partial n}\right|_{z=-h}=\epsilon^{2} \frac{\left(h_{t}+u h_{x}\right)}{\left(1+h_{y}^{2}\right)^{\frac{1}{2}}}, \\
\left.\frac{\partial \phi}{\partial y}\right|_{y- \pm \hat{b}}= \pm \epsilon^{2}\left(\hat{b}_{t}+u \hat{b}_{x}\right),
\end{gathered}
$$


where $n$ is the outward unit normal to the boundary curve in the cross-flow plane.

With $A_{0}(x)=O(1), A_{\mathrm{d}}(x, t)$ and $A_{5}(x, t)=O(\alpha)$ by assumption, it is clear that $\bar{u}=O(\alpha)$ by (25), whereas $v$ and $w$ are of $O(\alpha \epsilon)$ by (29)-(32) provided that $h_{t}, b_{t}=O(\alpha)$, $h_{x}, b_{x}=O(\alpha)$, as assumed. However, $h_{y}$, the section slope of the channel boundary, need not be limited to certain bounds, such as for vertical walls.

In accordance with the scaling, $\phi$ and $\zeta$ assume the following expansions :

$$
\begin{gathered}
\phi=\alpha\left[\phi_{1}(x, t)+\epsilon^{2} \phi_{2}(x, y, z, t)+O\left(\epsilon^{4}\right)\right], \\
\zeta=\alpha\left[\zeta_{1}(x, t)+\epsilon^{2} \zeta_{2}(x, y, t)+O\left(\epsilon^{4}\right)\right],
\end{gathered}
$$

with the velocity components $u=\phi_{x}, v=\phi_{y} / \epsilon, w=\phi_{z} / \epsilon$, all scaled by $c_{0}=\left(g h_{\mathrm{c}}\right)^{\frac{1}{2}}$. From this we see that $u-\bar{u}=O\left(\alpha \epsilon^{2}\right), v-\bar{v}=O(\alpha \epsilon), w-\bar{w}=O(\alpha \epsilon)$, and hence

$$
\begin{gathered}
\overline{u u}-\bar{u} \bar{u}=O\left(\alpha^{2} \epsilon^{4}\right), \\
p_{\mathrm{e}}-\overline{p_{\mathrm{e}}}=O\left(\alpha \epsilon^{2}, \alpha^{2}\right) .
\end{gathered}
$$

The last relation for the excess pressure $p_{\mathrm{e}}=p+z$ is valid throughout the depth and gives a measure of $\left|\partial p_{\mathrm{e}} / \partial z\right|=O\left(\alpha \epsilon^{2}\right)$ for the assumption of the pressure being hydrostatic as generally adopted in classical shallow-water long-wave theory. In particular, at the free surface, (36) becomes $\left|\left(p_{\mathrm{a}}+\zeta\right)-\left(\tilde{p}_{\mathrm{a}}+\tilde{\zeta}\right)\right|=O\left(\alpha \epsilon^{2}\right)$; hence if $\left|p_{\mathrm{a}}-\tilde{p}_{\mathrm{a}}\right| \leqslant O\left(\alpha \epsilon^{2}\right)$, then $|\zeta-\tilde{\zeta}|=O\left(\alpha \epsilon^{2}\right)$, indicating that the transverse variations of $\zeta$ from its transverse surface mean is always of higher order than $|\zeta|$, which is of $O(\alpha)$.

Thus, use of (35) resolves the term $\overline{u u}$ in (13). For the closure of (25) and (13), it remains to seek an expression for $\overline{p_{x}}$ in terms of $\bar{u}$ and $\tilde{\zeta}$. This is accomplished by evaluating the difference between the sectional free-surface mean and the section mean of the $x$-gradient of the Bernoulli equation, which yields the result

where

$$
\begin{gathered}
\overline{p_{x}}=\widetilde{p_{\mathrm{a} x}}+\widetilde{\zeta_{x}}+\epsilon^{2} \psi(x, t)+O\left(\alpha \epsilon^{4}\right), \\
\psi(x, t)=\alpha\left(\left.\widetilde{\phi_{2 x t}}\right|_{z=0}-\overline{\phi_{2 x t}}\right) .
\end{gathered}
$$

The term $\psi(x, t)$ in (37) depends on the solution of $\phi_{2}(x, y, z, t)$ which is the secondorder term in the expansion (33) for $\phi$ and which can be solved explicitly in terms of $\bar{u}$ and $\tilde{\zeta}$ once the specific geometry of the cross-section is given. This 'cross-flow' boundary-value problem for $\phi_{2}$ is prescribed by

$$
\begin{aligned}
\phi_{2 y y}+\phi_{2 z z}=-u_{1 x} & \left(y, z \in S_{\mathrm{c}}(x, t)\right), \\
\frac{\partial \phi_{2}}{\partial z}=\zeta_{1 t} & (z=0), \\
\frac{\partial \phi_{2}}{\partial n}=\left(\frac{1}{\alpha} h_{t}+u_{1} h_{x}\right) /\left(1+h_{y}^{2}\right)^{\frac{1}{2}} & (z=-h(x, y, t)), \\
\frac{\partial \phi_{2}}{\partial y}= \pm\left(\frac{1}{\alpha} \hat{b}_{t}+u_{1} \hat{b}_{x}\right) & (y= \pm \hat{b}(x, t)),
\end{aligned}
$$

where $S_{\mathrm{c}}$ denotes the cross-sectional flow region and $u_{1}=\phi_{1 x}$. This Neumann problem for the leading-order cross-flow velocity potential $\phi_{2}$ has a unique solution (up to an integral constant function of $(x, t)$ ) since the boundary conditions are easily seen to satisfy the solvability condition

$$
\oint_{C} \frac{\partial \phi_{2}}{\partial n} \mathrm{~d} s=-A(x, t) u_{1 x}
$$


$C$ being the boundary curve of $S_{c}(x, t)$. The solution $\phi_{2}$ depends on $\alpha u_{1} \approx \bar{u}(x, t)$ (as a parameter) in addition to the geometric configuration of the boundary.

Finally, substituting (35) and (37) into (13) yields with (25) the set

$$
\begin{gathered}
(2 b \tilde{\zeta})_{t}+\left[\left(A_{h}+2 b \tilde{\zeta}\right) \bar{u}\right]_{x}=-\left(A_{h}\right)_{t}, \\
\bar{u}_{t}+\bar{u}_{x}+\tilde{\zeta}_{x}+\epsilon^{2} \psi(x, t)=-\tilde{p}_{a x},
\end{gathered}
$$

where $\psi$ is given by (38). Equation (43) is exact and (44) has an error of $O\left(\alpha \epsilon^{2}\right)$ relative to the leading terms $\bar{u}_{t}$ and $\tilde{\zeta}_{x}$. The set of equations (43) and (44) is our generalized channel Boussinesq-class two-equation model in the general form which admits external forcing disturbances. It is applicable to weakly nonlinear, weakly dispersive and weakly forced long waves propagating, possibly in both directions, in a channel of arbitrary, non-uniform and varying cross-section. The forcing functions can be a submerged topography or a free-surface pressure distribution oscillating and moving along the channel.

\subsection{The channel Korteweg-de Vries model}

With additional assumptions that the main waves are unidirectional and the external forcing is sustained nearly at resonance of the system, the Boussinesq twoequation model (43) and (44) can be further reduced to become the so-called channel Korteweg-de Vries (cKdV) equation.

With the channel width and the mean water depth decomposed as

$$
b(x, t)=b_{0}(x)-b_{\mathrm{d}}(x, t), \quad \tilde{h}(x, t)=\tilde{h}_{0}(x)-\tilde{h}_{\mathrm{d}}(x, t),
$$

where $b_{0}(x)$ and $\tilde{h}_{0}(x)=A_{0}(x) / 2 b_{0}(x)$ represent the fixed components to which small unsteady perturbations $b_{\mathrm{d}}(x, t)$ and $\tilde{h}_{\mathrm{d}}(x, t)$ are superposed as forcing functions (taken positive if they reduce the flow cross-sectional area and negative otherwise), we adopt the multiscale transformation (see Johnson 1973) to give the new independent variables

$$
\xi=\int^{x} \frac{\mathrm{d} x}{c(x)}-t, \quad X=\epsilon^{2} x .
$$

In this coordinate system, the variations in the linearized wave speed $c(x)$, in depth $\tilde{h}_{0}(x)$ and in width $b_{0}(x)$ become functions of $X$ only, signifying their slow variation. By assuming for $f=\bar{u}, \tilde{\zeta}, \widetilde{p_{\mathrm{a}}}, b_{\mathrm{d}}, h_{\mathrm{d}}, A_{\mathrm{d}}$ and $\psi$ the expansion

$$
f(x, t ; \epsilon)=\epsilon^{2} f_{1}(\xi, X)+\epsilon^{4} f_{2}(\xi, X)+O\left(\epsilon^{6}\right),
$$

it can be shown (see Teng \& Wu 1990) that

$$
\begin{gathered}
\zeta_{1}=c u_{1} \quad\left(c= \pm\left[h_{0}(x)\right]^{\frac{1}{2}}\right), \\
A_{\mathrm{d} 1}=p_{\mathrm{a} 1}=0,
\end{gathered}
$$

where the sign $+($ or -$)$ is for right (or left)-going waves, and that $\zeta_{1}$ of a right-going waves satisfies

$$
c \zeta_{1 X}+\frac{3}{2} \frac{1}{h_{0}} \zeta_{1} \zeta_{1 \xi}+\frac{1}{2} h_{0}^{\frac{1}{2}} \psi_{1}+\frac{1}{4} h_{0}^{\frac{1}{2}}\left(\ln h_{0} b_{0}^{2}\right)_{X} \zeta_{1}=-\frac{1}{2}\left(\frac{1}{2 b_{0}} A_{\mathrm{d} 2}+p_{\mathrm{a} 2}\right)_{\xi}
$$

In addition, we have the following relationship between $u_{2}$ and $\zeta_{1}, \zeta_{2}$ :

$$
c u_{2}=\zeta_{2}-\frac{1}{4} \frac{1}{h_{0}} \zeta_{1}^{2}+\frac{1}{2} h_{0}^{\frac{1}{2}} \int_{\xi} \psi_{1} \mathrm{~d} \xi-\frac{1}{4} \frac{1}{b_{0}} A_{\mathrm{d} 2}+\frac{1}{2} p_{\mathrm{a} 2}-\frac{1}{4} h_{0}^{\frac{1}{2}}\left(\ln h_{0} b_{0}^{2}\right)_{X} \int_{\xi} \zeta_{1} \mathrm{~d} \xi
$$


Equation (49) is the generalized $\mathrm{KdV}$ equation expressed in terms of the stretched coordinates. Finally, restoring the original variables $\zeta$ and $u$ (standing for $\tilde{\zeta}$ and $\bar{u}$ ) in terms of the original coordinates $(x, t)$ for the laboratory system in which the fluid is at rest at infinity, we obtain the channel $K d V$ equation as

$$
\begin{aligned}
& \pm \frac{1}{c} \zeta_{t}+\zeta_{x}+\frac{3}{2} \frac{1}{h_{0}} \zeta \zeta_{x}+\frac{1}{2} \epsilon^{2} \psi+\frac{1}{4}\left(\ln h_{0} b_{0}^{2}\right)_{x} \zeta=-\frac{1}{2}\left(\frac{1}{2 b_{0}} A_{\mathrm{d}}+p_{\mathrm{a}}\right)_{x}, \\
& c u=\zeta-\frac{1}{4} \frac{1}{h_{0}} \zeta^{2}+\frac{1}{2} \epsilon^{2} \int \psi \mathrm{d} x-\frac{1}{4} \frac{1}{b_{0}} A_{\mathrm{d}}+\frac{1}{2} p_{\mathrm{a}}-\frac{1}{4}\left(\ln h_{0} b_{0}^{2}\right)_{x} \int_{x_{0}}^{x} \zeta \mathrm{d} x,
\end{aligned}
$$

where the + (or - ) sign holds for the case of the right-going (or left-going) waves and $x_{0}$ is some station at which $\zeta, u$ and all disturbances vanish.

With $\phi_{2}$ and hence $\psi$ assumed determined (in terms of $u$ and $\zeta$ ), equation (51) then provides the channel KdV-type one-equation model in the general form. For various special cases, this channel KdV equation agrees with the forced $\mathrm{KdV}$ model of $\mathrm{Wu}$ (1987) and Lee, Yates \& Wu (1989) for uniform rectangular channels, and with Shuto (1974) for rectangular channels of variable depth and width, without forcing.

\section{Cross-flows for some specific channel shapes}

There are four specific channel shapes for which solutions to the Neumann problem of the Poisson equation (39)-(42) can be obtained in closed form, three of which have been given by Teng (1990) and Teng \& Wu (1990). We cite the three and add here the solution for the variable parabolic channel.

\subsection{Variable rectangular channel}

The variable rectangular channel has its sidewalls at

$$
y= \pm b(x, t)= \pm\left[b_{0}(x)-b_{\mathrm{d}}(x, t)\right]
$$

and its bottom at $z=-h(x, t)=-\left[h_{0}(x)-h_{\mathrm{d}}(x, t)\right]$ (see figure $2 a$ ). The fixed depth $h_{0}(x)$ and width $b_{0}(x)$ may have small forcing disturbances $h_{\mathrm{d}}(x, t)$ and $b_{\mathrm{d}}(x, t)$ superposed which move along the bottom and the walls of the channel, respectively, keeping the instantaneous channel cross-section everywhere rectangular.

The corresponding cross-flow problem for $\phi_{2}$ has the following description :

$$
\begin{gathered}
\phi_{2 y y}+\phi_{2 z z}=-u_{1 x}(x, t) \quad(-h(x, t)<z<0, \quad|y|<b(x, t)), \\
\frac{\partial \phi_{2}}{\partial z}=\zeta_{1 t} \quad(z=0,|y|<b(x, t)), \\
\frac{\partial \phi_{2}}{\partial z}=-\mathrm{D} h \quad(z=-h(x, t)) \\
\frac{\partial \phi_{2}}{\partial y}= \pm \mathrm{D} b \quad(y= \pm b(x, t)),
\end{gathered}
$$

where $\mathrm{D} \equiv(1 / \alpha) \partial_{t}+u_{1} \partial_{x}$. (For the last condition, see (6).) The solution is

$$
\phi_{2}(x, y, z, t)=a_{0}(x, t)+z a_{1}(x, t)+a_{2}(x, t)\left(y^{2}-z^{2}\right)-\frac{1}{2} z^{2} u_{1 x},
$$



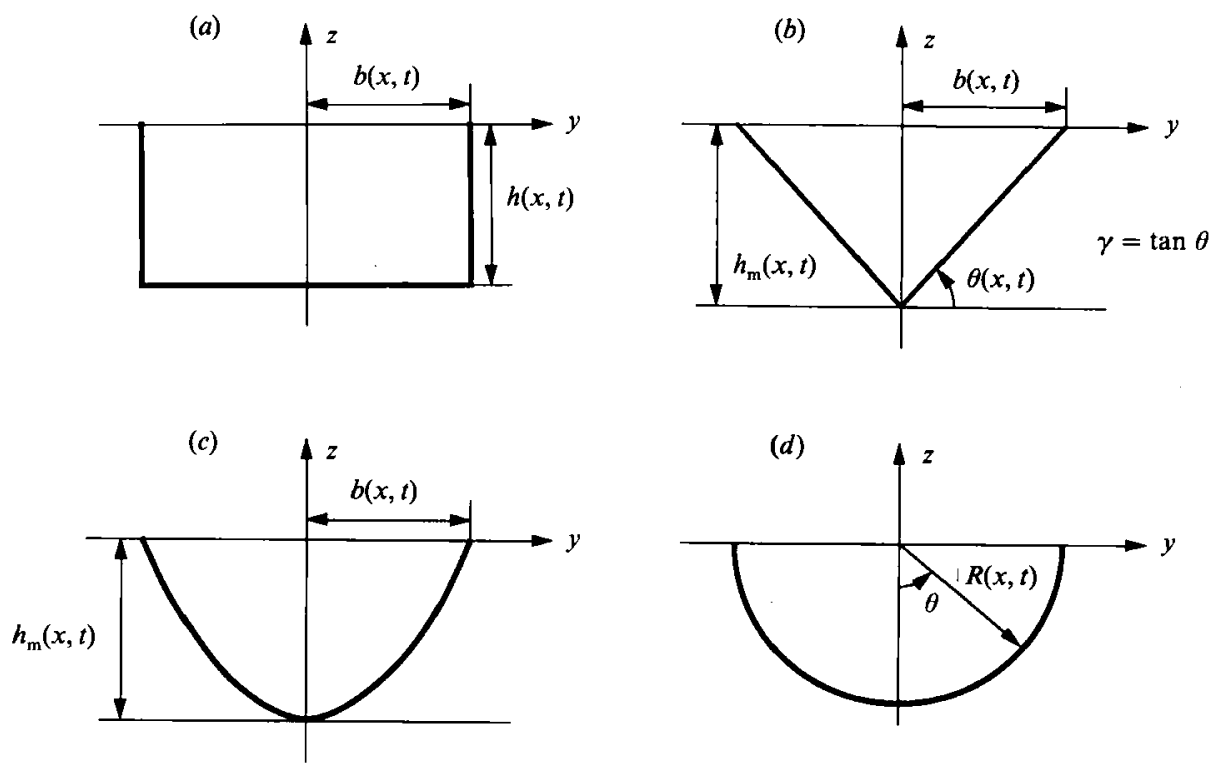

FIGURe 2. Four specific channel shapes: with $(a)$ rectangular, $(b)$ triangular, $(c)$ parabolic, and (d) semicircular cross-sections.

where

$$
\begin{gathered}
a_{1}(x, t)=\zeta_{1 t}=-\mathrm{D} h-2 a_{2} h-h u_{1 x}, \\
a_{2}(x, t)=\frac{1}{2} \mathrm{D} \ln b,
\end{gathered}
$$

and the undetermined function $a_{0}(x, t)$ represents a complementary solution.

From this $\phi_{2}$, we deduce that

$$
\begin{aligned}
\psi(x, t) & =\alpha\left(\widetilde{\phi_{2 x t}}-\overline{\phi_{2 x t}}\right) \\
& =\frac{1}{2} h \zeta_{x t t}+\frac{1}{6} h^{2} u_{x x t}+\frac{1}{8} h^{2} Q_{x t},
\end{aligned}
$$

where $Q=\alpha \mathrm{D} \ln b$, and we note that the additive term $a_{0}(x, t)$ in (53) for $\phi_{2}$ makes no contribution to $\psi$. Here, the two relations for $a_{1}$ in (54) are equivalent by virtue of the solvability condition. With the $\psi(x, t)$ determined, (43) and (44) completes the $\mathrm{gcB}$ model for the case of a rectangular channel.

For the cKdV model, the above result (56) for $\psi(x, t)$ further simplifies for the unidirectional motion and weak forcing (of order $O\left(\alpha \epsilon^{2}\right)$ ), so that $\zeta_{t}=-c \zeta_{x}, u_{t}=-\zeta_{x}$, which gives

$$
\psi=\frac{1}{3} h_{0}^{2} \zeta_{x x x}
$$

and the corresponding $\mathrm{cKdV}$ equation becomes

$$
\pm \frac{1}{c} \zeta_{t}+\zeta_{x}+\frac{3}{2} \frac{1}{h_{0}} \zeta \zeta_{x}+\frac{1}{6} \epsilon^{2} h_{0}^{2} \zeta_{x x x}+\frac{1}{4}\left(\ln h_{0} b_{0}^{2}\right)_{x} \zeta=-\frac{1}{2}\left(\frac{1}{2 b_{0}} A_{\mathrm{d}}+p_{\mathrm{a}}\right)_{x}
$$

\subsection{Variable triangular channel}

The triangular channel is bounded by two inclined plane walls situated at

$$
z+\left(h_{\mathrm{m}}-\gamma|y|\right)=0 \quad\left(|y| \leqslant h_{\mathrm{m}} / \gamma=b\right)
$$


where $h_{\mathrm{m}}$ and $\gamma$ may vary with $x$ and $t$ (see figure $2 b$ ). The cross-flow potential $\phi_{2}$ satisfies the Poisson equation (39) and the following boundary conditions:

$$
\begin{gathered}
\frac{\partial \phi_{2}}{\partial z}=\zeta_{1 t} \quad(z=0,|y| \leqslant b(x, t)) \\
\gamma \frac{\partial \phi_{2}}{\partial y} \operatorname{sgn} y-\frac{\partial \phi_{2}}{\partial z}=\mathrm{D} h_{\mathrm{m}}-|y| \mathrm{D} \gamma \quad\left(z=-h_{\mathrm{m}}+\gamma|y|,|y|<b(x, t)\right) .
\end{gathered}
$$

The solution is given by (53), now for the triangular channel with

$$
\begin{gathered}
a_{1}(x, t)=\zeta_{1 t}=-2 \mathrm{D} \tilde{h}-\tilde{h} u_{1 x}+\tilde{h} \mathrm{D} \ln \gamma, \\
a_{2}(x, t)=-\frac{1}{4} u_{1 x}-\frac{1}{4} \mathrm{D} \ln \gamma,
\end{gathered}
$$

where the mean depth $\tilde{h}$ of the triangular channel is

$$
\tilde{h}=\frac{A}{2 b}=\frac{1}{2} h_{\mathrm{m}}
$$

This solution for $\phi_{2}$ gives, by (38),

where $q=\alpha \mathrm{D} \ln \gamma$.

$$
\psi=\frac{2}{3} \tilde{h} \zeta_{x t t}+\frac{1}{6}\left(\tilde{h}^{2}-\frac{1}{4} b^{2}\right) u_{x x t}-\frac{1}{6}\left(\tilde{h}^{2}+\frac{1}{4} b^{2}\right) q_{x t},
$$

For the $\mathrm{cKdV}$ equation for right-going waves, the above $\psi$ reduces to

$$
\psi=\frac{1}{2}\left(h_{0}^{2}+\frac{1}{12} b_{0}^{2}\right) \zeta_{x x x}
$$

where $h_{0}=\tilde{h}_{0}$ (see (45)). The $\psi(x, t)$ given by $(63)$ completes the gcB model and the $\psi$ of (64) affords the corresponding $\mathrm{cKdV}$ model for variable triangular channels.

\subsection{Variable parabolic channel}

For a channel with a parabolic cross-section (see figure $2 c$ )

$$
z=-h(x, y, t)=h_{\mathrm{m}}\left(\frac{y^{2}}{b^{2}}-1\right),
$$

where $h_{\mathrm{m}}(x, t)$ and $b(x, t)$ are the maximum depth and the maximum half-width of the channel, $\phi_{2}$ satisfies (39) and the following boundary conditions

$$
\begin{gathered}
\frac{\partial \phi_{2}}{\partial z}=\zeta_{1 t} \quad(z=0) \\
\frac{\partial \phi_{2}}{\partial z}=-\mathrm{D} h-\frac{\partial \phi_{2}}{\partial y} h_{y} \quad(z=-h(x, y, t)) .
\end{gathered}
$$

The solution for $\phi_{2}$ is again of the form (53), now with

$$
\begin{gathered}
a_{1}(x, t)=\zeta_{1 t}=-\frac{3}{2} \mathrm{D} \tilde{h}-\tilde{h} u_{1 x}+\frac{\tilde{h}}{2 \alpha} \mu, \\
a_{2}(x, t)=-\frac{1}{6} u_{1 x}-\frac{1}{6 \alpha} \mu, \\
\tilde{h}=\frac{A}{2 b}=\frac{2}{3} h_{\mathrm{m}},
\end{gathered}
$$


where $\mu=\alpha \mathrm{D} \ln \left(\tilde{h} / b^{2}\right)$. From this solution of $\phi_{2}$ we obtain

$$
\psi(x, t)=\frac{9}{5} \tilde{h} \zeta_{x t t}+\left(\frac{6 \tilde{h}^{2}}{35}-\frac{b^{2}}{45}\right) u_{x x t}-\left(\frac{3 \tilde{h}^{2}}{35}+\frac{b^{2}}{45}\right) \mu_{x t}
$$

The leading-order term of $\psi$ reduces for the right-going waves to

$$
\psi(x, t)=\left(\frac{3}{7} h_{0}^{2}+\frac{b_{0}^{2}}{45}\right) \zeta_{x x x}
$$

\subsection{Variable semicircular channel}

For a channel with semicircular cross-section of variable radius $R(x, t)$ (see figure $2 d$ ), we employ the cylindrical coordinates $(x, r, \theta)$, with $z=-r \cos \theta, y=r \sin \theta$ so that the boundary-value problem is prescribed as

$$
\begin{gathered}
\left(\frac{\partial^{2}}{\partial r^{2}}+\frac{1}{r} \frac{\partial}{\partial r}+\frac{1}{r^{2}} \frac{\partial^{2}}{\partial \theta^{2}}\right) \phi_{2}=-u_{1 x} \quad\left(0 \leqslant r \leqslant R,|\theta|<\frac{1}{2} \pi\right) \\
\frac{1}{r} \frac{\partial \phi_{2}}{\partial \theta}= \pm \zeta_{1 t} \quad\left(\theta= \pm \frac{1}{2} \pi, 0 \leqslant r \leqslant R\right) \\
\frac{\partial \phi_{2}}{\partial r}=\mathrm{DR} \quad\left(r=R(x, t),|\theta|<\frac{1}{2} \pi\right)
\end{gathered}
$$

and the solvability condition gives

$$
\oint_{C} \frac{\partial \phi_{2}}{\partial n} \mathrm{~d} s=\pi R \mathrm{D} R+2 R \zeta_{1 t}=-\frac{1}{2} \pi R^{2} u_{1 x}
$$

The solution of this problem is

$$
\begin{gathered}
\phi_{2}=\phi_{\mathrm{p}}+\phi_{\mathrm{c}} \\
\phi_{\mathrm{p}}=-r a_{1}(x, t) \cos \theta-\frac{1}{4} r^{2} u_{1 x}, \\
a_{1}(x, t)=\zeta_{1 t}=-\frac{1}{2} \pi \mathrm{D} R-\frac{1}{4} \pi R u_{1 x}, \\
\phi_{\mathrm{c}}=a_{0}(x, t)+\frac{a_{1}}{\pi} R \Phi \\
F(\xi)=\Phi+\mathrm{i} \Psi=2+\frac{1}{\xi}(1-\xi)^{2} \ln (1-\xi)-\frac{1}{\xi}(1+\xi)^{2} \ln (1+\xi),
\end{gathered}
$$

where $\Phi$ and $\Psi$ are the real and imaginary part of $F$ and

$$
\xi=\frac{1}{R}(y+\mathrm{i} z)=\frac{r}{R} \mathrm{e}^{\mathrm{i} \theta-\mathrm{i} \pi / 2} .
$$

Here $\phi_{\mathrm{p}}$ stands for a particular solution, $\phi_{\mathrm{c}}$ the complementary solution and $a_{0}(x, t)$ is an arbitrary function. From the above solution of $\phi_{2}$, we deduce the result

$$
\psi(x, t)=\frac{\tilde{h}}{\pi^{2}}\left(\frac{76}{3}-16 \ln 2-\pi^{2}\right) \zeta_{x t t}+\frac{2}{3 \pi^{2}} \tilde{h}^{2} u_{x x t}
$$

in which the higher-order terms are neglected, and $R$ has been converted to $\tilde{h}=\frac{1}{4} \pi R$. 
For the cKdV equation, we have

$$
\psi(x, t)=\frac{h_{0}^{2}}{\pi^{2}}\left(\frac{74}{3}-16 \ln 2-\pi^{2}\right) \zeta_{x x x}=0.3756 h_{0}^{2} \zeta_{x x x} .
$$

For the general case of arbitrary cross-sectional shape, we may apply various methods available in potential theory, such as Green's function, the conformal mapping technique, etc. to derive the $\mathrm{gcB}$ and the $\mathrm{cKdV}$ models for a given geometry.

\subsection{Rescaling of the wave models}

Our long wave models are written in terms of variables scaled horizontally by a typical wavelength $\lambda$ and vertically and transversally by the unperturbed mean water depth $h_{\mathrm{c}}$. This different scaling is essential in deriving the wave models by applying a perturbation technique. However, after the models are obtained with all terms in the equations properly balanced, it will be more convenient to rescale all quantities based on one single scale, namely the unperturbed mean water depth $h_{\mathrm{c}}$, since the typical wavelength $\lambda$ is an unknown itself (aside from being much greater than $h_{\mathrm{c}}$ ). After this rescaling, the small parameter $\epsilon$ no longer appears in the equations.

\section{Solitary and cnoidal waves in uniform channels}

Of particular interest is the special case of uniform channels, each with a specific yet arbitrary and fixed cross-sectional shape, for the uniformity of the medium must imply existence of waves of permanent form which merits study. For this case, with $b_{0}=$ const., $h=$ const., the gcB equations become

$$
\begin{gathered}
\zeta_{t}+[(h+\zeta) u]_{x}=B_{t}, \\
u_{t}+u u_{x}+\zeta_{x}-\frac{1}{3} \kappa^{2} h^{2} u_{x x t}=-p_{\mathrm{a} x},
\end{gathered}
$$

where $\zeta$ stands for $\tilde{\zeta}, u$ for $\bar{u}, h$ for $\tilde{h}_{\mathrm{0}}=A_{h} / 2 b=$ const., and $B(x, t) \equiv A_{\mathrm{d}} / 2 b$, with $A_{\mathrm{d}}$ being the so-called blockage ratio. In (74), the dispersion term with $u_{x x t}$ is obtained from the original expression after using the first-order approximation $\zeta_{t}=-h u_{x}$ in converting the third-order $\zeta$-derivative terms without affecting the error estimate, so that for any uniform channel

$$
\psi=-\frac{1}{3} \kappa^{2} h^{2} u_{x x t}
$$

where $\kappa^{2}$ is the only factor in (73) and (74) that depends explicitly on the channel cross-sectional shape. In particular, for the four specific channel shapes, with $b_{0}=h$ for the parabolic and triangular channels, we have the values of $\kappa$ shown in table 1 . With $\kappa=1$ for the rectangular channel as the standard, the value of $\kappa$ appears to increase the further the channel shape departs from the rectangular form. Theoretically, the dispersion term $\psi$ in (75) may be replaced by $\frac{1}{3} \kappa^{2} h^{2} \zeta_{x x x}$ or $\frac{1}{3} \kappa^{2} h \zeta_{x t t}$, since they are all asymptotically equivalent to each other, but the form (75) is preferred, its advantage in numerical stability being a primary reason.

The corresponding cKdV equation is readily deduced from (51) as

$$
\pm \frac{1}{h^{\frac{1}{2}}} \zeta_{t}+\zeta_{x}+\frac{3}{2} \frac{1}{h} \zeta \zeta_{x}+\frac{1}{6} \kappa^{2} h^{2} \zeta_{x x x}=-\frac{1}{2} P_{x}
$$

where $P=p_{\mathrm{a}}+B$, which shows, for this theoretical model, the equivalence between the surface pressure and the submerged blockage as forcing agencies. 


$\begin{array}{cc}\text { Uniform channel shape } & \text { Shape factor } \kappa \\ \text { rectangular } & 1 \\ \text { semicircular } & 1.06 \\ \text { parabolic } & 1.16 \\ \text { triangular } & 1.27\end{array}$

TABLE 1. Uniform channel shapes and their corresponding $\kappa$-values

It is quite evident that the uniformity of the medium must imply existence of waves which remain permanent in form as they propagate, even though the water depth may vary across the channel, because the only parameters characterizing the channel geometry are the section-mean depth $h$ and the channel shape factor $\kappa$, both being constant for uniform channels. In fact, both the gcB and $\mathrm{cKdV}$ equations for a ' $\kappa$-shaped' uniform channel (designated with a specific value of $\kappa$ ) are reduced to that for the rectangular channel under the similarity transformation:

$$
x=\kappa x^{\prime}, \quad t=\kappa t^{\prime}, \quad h=h^{\prime}, \quad \zeta=\zeta^{\prime}, \quad u=u^{\prime}, \quad B=B^{\prime}, \quad p_{\mathrm{a}}=p_{\mathrm{a}}^{\prime},
$$

with the primed quantities being referred to the analogous rectangular channel. A similar transformation was given by Peregrine $(1968)$; $(77)$ is however simpler and more general with forcing terms. This result is obvious; and we therefore have the following uniform-channel analogy theorem (proposed by Wu):

Theorem. If the set $u=\hat{u}(x, t ; h), \zeta=\hat{\zeta}(x, t ; h)$ is the solution to the gcB equations or the $c K d V$ equations for a rectangular channel of depth $h$, then for a specific $\kappa$-shaped uniform channel modelled by the same equations, the solution is $u=\hat{u}(x / \kappa, t / \kappa ; h)$, $\zeta=\hat{\zeta}(x / \kappa, t / \kappa ; h)$ for the same mean water depth $h$, provided the forcing distribution, if any, and the initial values also satisfy the relations (77).

This theorem is obvious in consequence of (77). The corresponding forcing distributions and initial values so defined (by transformation (77)) for the $\kappa$-shaped and the corresponding channels will be said to be analogous to each other. This uniform-channel analogy means that two analogous forcing distributions moving with the same velocity $U$ will produce waves of equal amplitude travelling with the same wave velocity $c$, since the arguments of the phase functions of the two sets of waves will be related by

$$
k(x-c t)=\kappa k\left(x^{\prime}-c t^{\prime}\right)=k^{\prime}\left(x^{\prime}-c t^{\prime}\right), \quad k^{\prime}=\kappa k,
$$

so that the wavenumber will be the only wave property that will be varied under the analogy, being converted from $k$ for the $\kappa$-shaped channel to $k^{\prime}=\kappa k$ for the analogous rectangular channel, or vice versa. Whence for a channel with $\kappa>1$, the waves are more gently sloped and more spread out than the analogous waves in the rectangular channel. With this analogy between channels, we therefore need only consider the rectangular channel, of which the theory is classical.

Without forcing, the $\mathrm{KdV}$ equation admits the known solitary wave

$$
\begin{gathered}
\zeta\left(x^{\prime}, t^{\prime} ; k^{\prime}\right)=\alpha \operatorname{sech}^{2}\left[k^{\prime}\left(x^{\prime}-c t^{\prime}\right)\right], \\
k^{\prime}=\left(\frac{3}{4} \alpha\right)^{\frac{1}{2}}, \quad c=1+\frac{1}{2} \alpha \quad(\alpha=a / h),
\end{gathered}
$$

where $a$ is the wave amplitude and the water depth $h(=1)$ is recovered for completeness in the expression for $\alpha$. For a $\kappa$-shaped uniform channel, with the same 
mean depth $h(=1)$, solitary waves exist, and, according to the above theorem, are the $\zeta(x, t ; k)$ as given by $(79 a)$, i.e.

$$
\begin{gathered}
\zeta(x, t ; k)=\alpha \operatorname{sech}^{2}[k(x-c t)], \\
\text { provided } \quad k=\left(\frac{3}{4} \alpha\right)^{\frac{1}{2}} \kappa^{-1}, \quad c=1+\frac{1}{2} \alpha \quad(\alpha=a / h) .
\end{gathered}
$$

Similarly, a $\kappa$-shaped channel can support cnoidal waves given by

$$
\begin{gathered}
\zeta=\alpha \mathrm{cn}^{2} k s, \quad s=(x-c t), \\
k=\kappa^{-1} \frac{(3 \beta)^{\frac{1}{2}}}{2 h}, \quad c=1+\frac{1}{2}(2 \alpha-\beta), \quad 0<\alpha<\beta,
\end{gathered}
$$

where the modulus of the Jacobian elliptic function cn $(k s \mid m)$ is

and

$$
\begin{gathered}
m=(\alpha / \beta)^{\frac{1}{2}}, \\
\lambda=\kappa \lambda^{\prime}=\frac{4 \kappa h}{(3 \beta)^{\frac{1}{2}}} K(m)
\end{gathered}
$$

is the wavelength of $\zeta, K(m)$ being the complete elliptic integral of the first kind. The above equation for $\lambda$ determines $\beta=\hat{\beta}(\kappa h / \lambda, \alpha)$. Therefore, for equal wave height $\alpha$ and dispersion parameter $\beta$, the cnoidal waves in a $\kappa$-shaped channel with a length $\kappa$ times that in the analogous rectangular channel, and $(81 b)$ shows that the wave slope is $k / k^{\prime}=1 / \kappa$ times that in the rectangular channel. For $\kappa=1$, this reduces to the classical result.

For the Boussinesq equations, there can be several first-approximation wave solutions, all belonging to the Boussinesq family; they arise from having the thirdorder derivative term in the equations modified by incorporating various first-order relations. In order to make comparisons with the $\mathrm{KdV}$ model, we proceed to find stationary solutions to the $\mathrm{gcB}$ equations (73) and (74) without forcing. For stationary waves, we seek solutions of the form

$$
\zeta=\zeta(s), \quad u / c=v(s), \quad s=(x-c t) / \kappa .
$$

Substituting (82) into (73) and (74), and integrating the equations once yields

$$
\begin{gathered}
-\zeta+(1+\zeta) v=0 \\
\frac{1}{3} v^{\prime \prime}+\frac{1}{2} v^{2}-v+\frac{1}{c^{2}} \zeta-C_{1}=0
\end{gathered}
$$

where the prime denotes $\mathrm{d} / \mathrm{d} s$, and $C_{1}$ is an integration constant. Using (83), we may expand $\zeta$ in (84) as

$$
\zeta=v /(1-v)=v+v^{2}+O\left(\alpha^{3}\right),
$$

which is consistent with the error estimate for (84) so, with a further integration,

$$
c^{2}\left(\frac{\mathrm{d} v}{\mathrm{~d} s}\right)^{2}=-\left(2+c^{2}\right) v^{3}+3\left(c^{2}-1\right) v^{2}+6 c^{2} C_{1} v+C_{2} \equiv c^{2} G(v ; c),
$$

where $C_{2}$ is an integration constant. For bounded solutions, the zeros of $G$ must be real, and bounded solutions must oscillate periodically between two of the zeros of $G$ where $G$ is positive.

For solitary waves, (86) has, with $C_{1}=C_{2}=0$, the integral

$$
u / c=v=\alpha \operatorname{sech}^{2}[k(x-c t) / \kappa] \text {, }
$$


where

$$
\begin{gathered}
c^{2}=1+\frac{1}{3}\left(2+c^{2}\right) \alpha \approx 1+\alpha, \\
k^{2}=\frac{1}{4}\left(1+2 / c^{2}\right) \alpha \approx \frac{3}{4} \alpha .
\end{gathered}
$$

Accordingly, we have for the wave elevation, to first order by the gcB model,

$$
\zeta=v /(1-v) \approx \alpha \operatorname{sech}^{2}[k(x-c t) / \kappa] .
$$

This solution of $\zeta$ for a $\kappa$-shaped channel modelled by the gcB equations reduces with $\kappa \rightarrow 1$ to the solitary wave of Boussinesq for rectangular channels, which we will designate $\mathrm{gB}(\mathrm{I})$.

For waves of finite length, $C_{1}$ and $C_{2}$ in (86) can be chosen such that

$$
\begin{gathered}
\left(\frac{\mathrm{d} v}{\mathrm{~d} s}\right)^{2}=\left(1+\frac{2}{c^{2}}\right) v(\alpha-v)(v+\beta-\alpha) \quad(0<\alpha<\beta), \\
c^{2}=[3+2(2 \alpha-\beta)] /[3-(2 \alpha-\beta)] .
\end{gathered}
$$

This equation has the integral

where

$$
\begin{gathered}
v=\alpha \mathrm{cn}^{2} k(x-c t), \\
k=\left[\left(2+c^{2}\right) \beta\right]^{\frac{1}{2}} / 2 \kappa c,
\end{gathered}
$$

which is a cnoidal wave in the $\kappa$-shaped channel, with wave height $\alpha$, velocity $c$, and length

$$
\lambda=(2 / k) K(m), \quad m=(\alpha / \beta)^{\frac{1}{2}},
$$

$K(m)$ being the complete elliptic integral of the first kind, and $m$ is also the argument of the elliptic function.

Alternatively, a solitary wave solution of the generalized Boussinesq equations can be obtained by integrating (84) after substituting

$$
\zeta=\frac{v}{1-v}
$$

by $(83)$, for $\zeta$ in $(84)$, giving

$$
\mathrm{d} v / \mathrm{d} s= \pm[G(v ; c)]^{\frac{1}{2}}
$$

with

$$
G(v ; c)=-v^{3}+3 v^{2}+\left(6 / c^{2}\right)[v+\ln (1-v)]+C_{1} v+C_{2},
$$

$C_{2}$ being an integration constant. For solitary waves, $v$ vanishes exponentially as $s \rightarrow \pm \infty$, hence $C_{1}=C_{2}=0$ so that $G \rightarrow 0$ like $v^{2}$ as $v \rightarrow 0$. In addition, the symmetry of a solitary wave implies

$$
\mathrm{d} v / \mathrm{d} s=0 \text { at } s=0,
$$

corresponding to which $G(v ; c)$ has a simple zero at $v=v_{\mathrm{c}}$, say, so that $G\left(v_{\mathrm{c}} ; c\right)=0$ determines $c$. Therefore, by $(92 b), v_{c}$ satisfies

$$
c^{2}=-6[v+\ln (1-v)] / v^{2}(3-v),
$$

which gives directly $c=c\left(v_{\mathrm{c}}\right)$. Thus we obtain the solitary wave solution of the gcB model for $v(s)$ by quadrature,

$$
s= \pm \int_{v_{\mathrm{c}}}^{v} \frac{\mathrm{d} v}{[G(v ; c)]^{\frac{1}{2}}}
$$

And accordingly we have the free-surface elevation $\zeta$ by (91), which we designate by gB.

A comparison between the solitary wave solutions given by the gcB and the $\mathrm{cKdV}$ models shows that $\mathrm{cKdV}$ - and $\mathrm{gB}(\mathrm{I})$-solitary waves have the same profile based on 

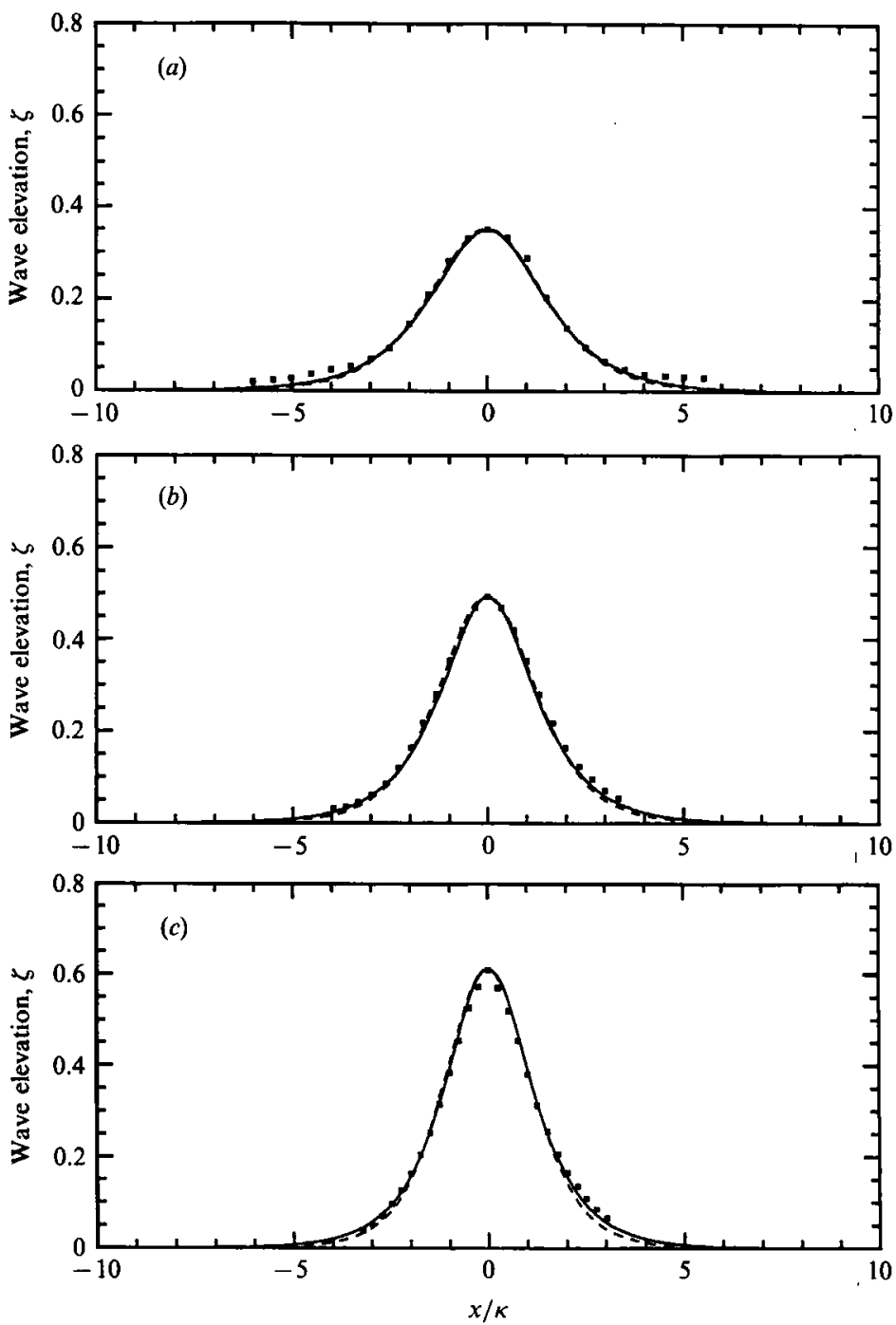

Figure 3. Profiles of solitary wave in a uniform channel of shape factor $\kappa$ (equation (77)) in the wave frame: $(a) \alpha=0.350,(b) \alpha=0.493,(c) \alpha=0.610$ : a, experiment, Daily \& Stephan (1952), for a rectangular channel;,$- \mathrm{gB} ;--, \mathrm{KdV}, \mathrm{gB}(\mathrm{I})$.

the same amplitude, while the $\mathrm{gB}$-solitary wave has a profile which is practically the same as the other two for small amplitudes, $\alpha<0.3$, and shows for $\alpha=0.5$ and 0.6 only a slight difference in having a little higher foot-hills than the cKdV soliton profile. These results are shown in figure $3(a-c)$ for $\alpha=0.350,0.493$ and 0.61 , together with the experimental results obtained by Daily \& Stephan (1952) for rectangular channels, i.e. with $\kappa=1$. In their comparative studies, Daily \& Stephan also included various other theoretical results. Of these theories, Boussinesq's profile (identical with the $\mathrm{KdV}$ profile) of the solitary wave is found to give the most consistent agreement with experiments. In the range $0.12<\alpha<0.61$ covered, the best agreement is reached at $\alpha=0.23$ and shows an almost complete coincidence between theory and experiment throughout the wave stretch. At higher $\alpha$, the theoretical profile exhibits a very slightly broader crest and narrower base than what 


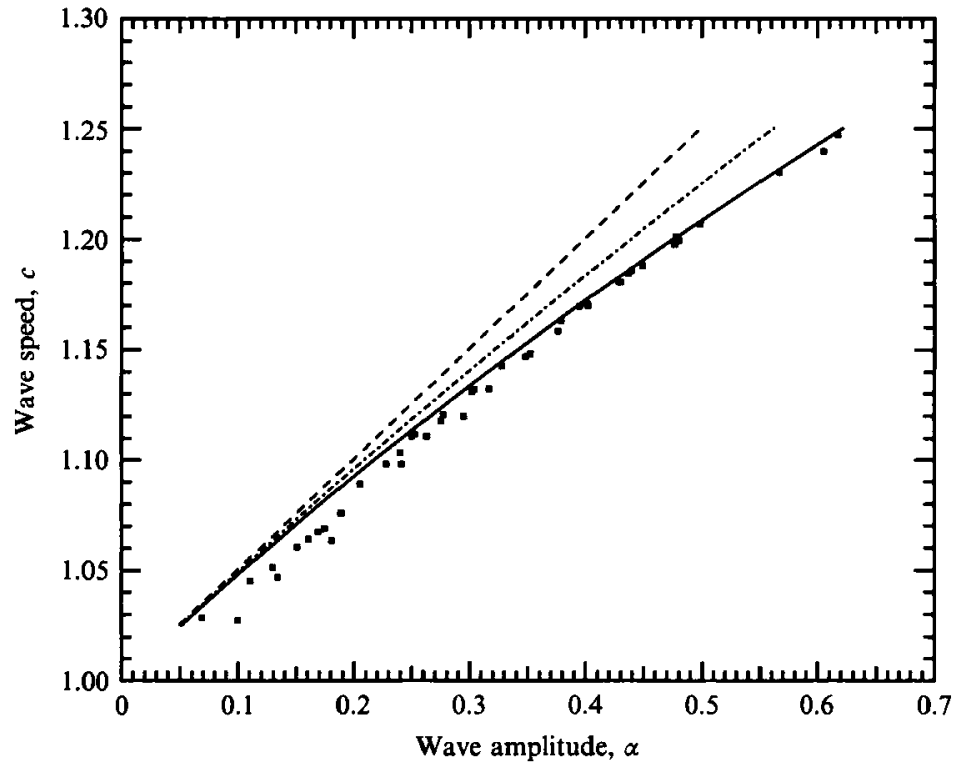

FIGURE 4. Wave speed $c$ versus wave amplitude $\alpha$ for solitary waves in uniform channels of arbitrary cross-sectional shape and equal mean depth : $a$, experiment, Daily \& Stephan (1952), for a rectangular channel; $\cdot-\cdot-\cdot$, Russell, $\mathrm{gB}(\mathrm{I}), c=(1+\alpha)^{\frac{1}{2}} ;---, \mathrm{KdV}, c=1+\frac{1}{2} \alpha ;-, \mathrm{gB}$.

was measured, and with a slight opposite trend at lower $\alpha$. The new profile of gB seems to improve slightly the agreement near the base, though not by a sufficient margin.

The theoretical results of wave velocities given by our two channel flow models are shown in figure 4 together with the experimental results of Daily \& Stephan (1952), who find that the wave velocity is adequately prescribed for all practical purposes by the relation $c=(1+\alpha)^{\frac{1}{2}}$ first derived empirically by Russell and theoretically by Boussinesq and Rayleigh. This theoretical value is about $2.5 \%$ greater than the experimental data at $\alpha=0.6$ and with percentile difference increasing at lower $\alpha$. The velocity given by the $c K d V$ model, $c=1+\frac{1}{2} \alpha$, gives a greater departure from the experimental data. In contrast, the wave velocity determined numerically by the gB solution (94) exhibits the best agreement, especially for higher $\alpha$. However, we cannot find concrete reasons for explaining this improvement.

In the comparative study made by Daily \& Stephan, the effects of viscous dissipation were included by using their empirical formula

$$
\left(\frac{a}{h}\right)^{-\frac{1}{4}}-\left(\frac{a_{0}}{h}\right)^{-\frac{1}{4}}=\frac{1}{20}\left(1-\frac{h}{b}\right)^{2}\left(\frac{\nu^{2}}{g h^{3}}\right)^{\frac{1}{h}} \frac{x}{h},
$$

where $a=a(x)$ is the wave amplitude at $x$ in water of $\operatorname{depth} h, a_{0}=a(0), b$ is the channel half-width, and $\nu$ the kinematic viscosity of water. This gives slightly lower attenuation rates than Keulegan's (1948) theory, and also Miles's (1976) theory. However, we note that any attenuation of a profile which is initially $\alpha \operatorname{sech}^{2} k x$ must induce departure from the purely $\operatorname{sech}^{2}(k x)$ profile, because the excess mass, being conserved on physical grounds, is but a one-parameter family in $\alpha$. In the literature, there do not seem to be any discussions of the effect due to changes from the $\operatorname{sech}^{2}(k x)$ profile on possible modifications of the dissipation rates of wave profiles. 

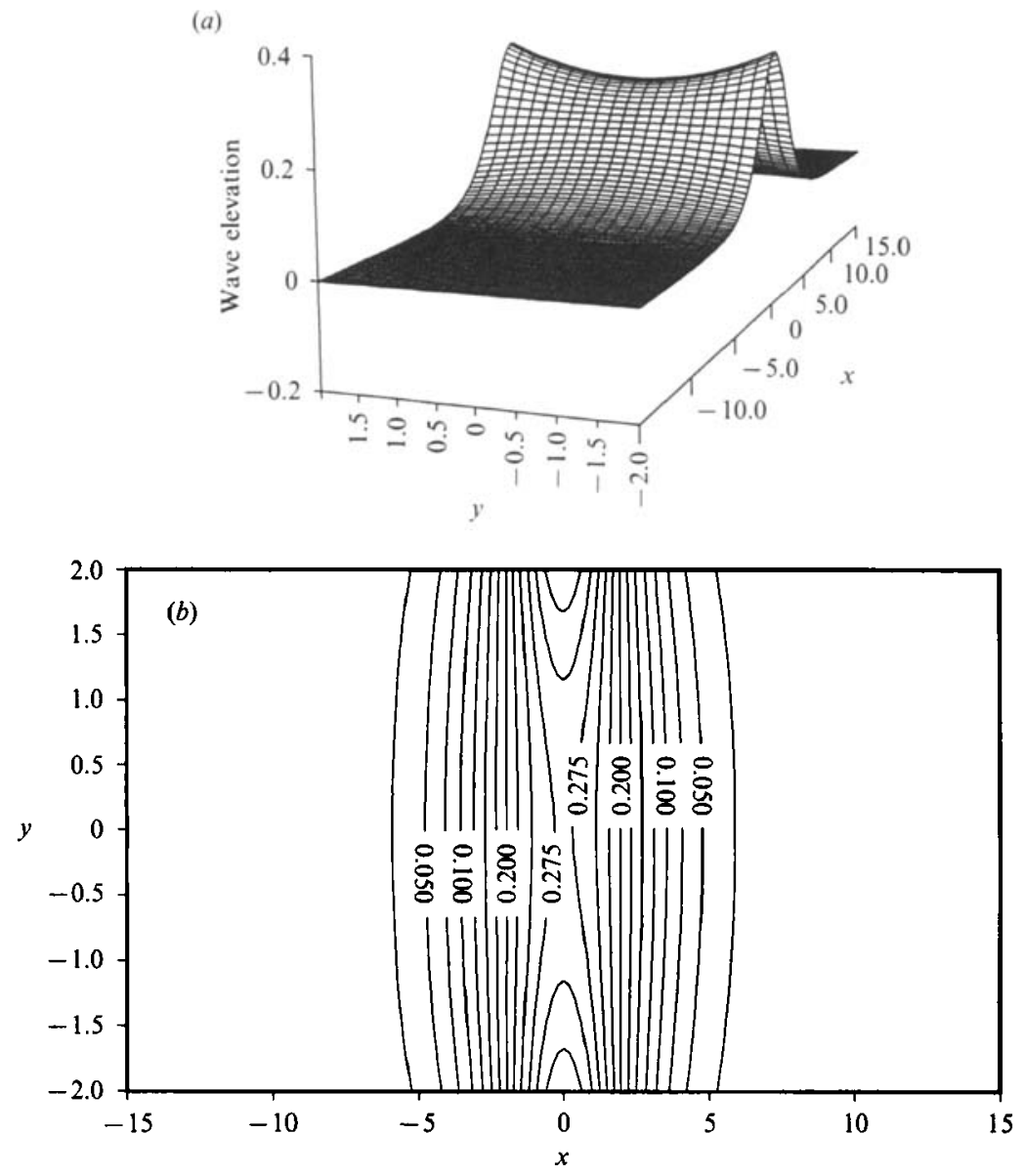

Fraure 5. A three-dimensional solitary wave based on the cKdV model, with section-mean amplitude of $\alpha=0.3$, in a uniform triangular channel of half-vertex angle of $45^{\circ}:(a)$ the perspective wave elevation, $(b)$ the elevation contour plot; $\Delta \zeta=0.025$ between consecutive curves.

For periodic waves of finite length in a $\kappa$-shaped channel, both models admit cnoidal waves, though the respective wave profiles have some differences based on equal $(\alpha, \beta)$ as both wavelength $\lambda$ and maximum wave slope $k \alpha$ have different functional dependence on $(\alpha, \beta)$, as does the wave velocity $c$ on $(\alpha, \beta)$ for the two models. However, we shall not pursue this comparison further here.

Another interesting feature of a solitary wave travelling in a non-rectangular uniform channel is that, unlike in the rectangular case for which the solitary wave maintains a profile uniformly across the channel, the wave amplitude in a nonrectangular channel varies across the width. This variation can be predicted by our two wave models. Figures $5(a)$ and $5(b)$ show the three-dimensional wave patterns of a solitary wave travelling in a triangular channel of half-vertex angle of $45^{\circ}$. The results are computed by first solving the cKdV equation to obtain the section-mean solitary wave solution (79) and (80) with $\kappa^{2}=2\left(h_{0}=1, b=2\right)$, and then substituting these first-order results into perturbation expansion (33) with cross-flow solutions $(53),(60)$ and $(61)$ to recover the three-dimensional wave field to the second order. By applying the Bernoulli equation at the free surface and using the first-order approximations $\zeta=c u$ and $\partial_{t}=-c \partial_{x}$, we have for the present case 


$$
\begin{aligned}
\zeta(x, y, t) & =-\phi_{t}-\frac{1}{2} \phi_{x}^{2}+O\left(\alpha^{2} \epsilon^{2}\right) \quad(z=0), \\
& =\tilde{\zeta}-\frac{1}{4} \tilde{\zeta}_{x x} y^{2}+a_{0}^{*}(x, t)+O\left(\alpha^{2} \epsilon^{2}\right) \quad(-b<y<b, b=2),
\end{aligned}
$$

where $\tilde{\zeta}$ represents the section-mean solution and $a_{0}^{*}(x, t)$ is determined upon taking the surface mean of $(96 a)$ to yield for $b=2$

$$
a_{0}^{*}(x, t)=\frac{1}{3} \tilde{\zeta}_{x x}+o\left(\alpha^{2}\right) \text {. }
$$

The results show that the wave crest varies across the channel in the form of a sideways saddle with its lowest elevation and widest spread occurring at the centreplane. Its profile along the crestline is similar to the curve given by Peregrine (1968).

\section{Discussion and conclusion}

We have derived two types of theoretical model - the generalized channel Boussinesq model (gcB, (43)-(44)) and the channel KdV model (cKdV, (51)) - for describing the generation and propagation of nonlinear long waves in a channel of variable cross-section which may vary both in space and time in addition to possibly being subject to external forcing excitations. They are derived from the inviscid Euler equations by using perturbation technique for quasi-one-dimensional long waves on a shallow layer of water.

Based on the underlying assumptions, we infer that the gcB model has a broader range of general validity than the $c K d V$ model. The magnitude of the perturbed quantities can be of $O(\alpha)$ for the gcB model whereas, for the cKdV model, the magnitude of the external forcing functions are required to be of the next higher order, i.e. of $O\left(\alpha^{2}\right)$ due to the limitation prevailing at resonance. Further, the gcB model allows bidirectional waves and is valid for a considerably larger range of Froude number. It permits the channel cross-sections to have more arbitrary variations in space and time. The cKdV model is best suitable for studying unidirectional wave motions with near resonant external forcing as originally prescribed. Under that premise, the $\mathrm{cKdV}$ model has the advantage over the gcB model that solutions are simpler to obtain and some theoretical points are more directly seen such as the net effect of external forcing functions or the mass and energy conservation laws.

The analysis and results presented in the previous section show that the specific cross-sectional configuration only affects the coefficients of the dispersion terms in the two models. In this respect, the $\mathrm{cKdV}$ model gives a slightly clearer picture on this particular effect, since in this case, a change in the cross-sectional shape only changes the coefficient of the single dispersion term $\zeta_{x x x}$ while the other terms are unaffected. Quantitatively, we may list the dispersion terms in the cKdV equations for uniform channels with rectangular, semicircular, parabolic and triangular crosssections (with $h_{0}=b$ for the last two cases) as given in table 1 . From these results we see that the mean slope of the channel sidewalls plays an explicit role in producing the dispersive effects : the greater the departure of the channel sectional shape from rectangular, the greater the dispersive effect. For uniform channels, we also enunciated a uniform-channel analogy theorem, which shows the analogy of wave solutions among different channel configurations.

Solitary waves can be generated by an external near-resonant forcing, and the cKdV model shows that a moving pressure distribution at the free surface and a floating or submerged moving object, like a ship, play an exactly equivalent role as 
forcing functions provided the magnitude of external forcing is small, of $O\left(\alpha^{2}\right)$. When external forcing becomes stronger in order of magnitude, the validity of the cKdV model becomes questionable, yet the gcB model is seen still capable of distinguishing between a free-surface pressure distribution and a submerged moving object as external forcing functions. For a semi-submerged moving object, the cKdV equation (51) shows that the effective strength of forcing is directly related to the blockage ratio of the disturbance's frontal projected area to the cross-sectional area, while the specific shape and the position in the $(y, z)$-plane of the disturbance have little effect on the long waves so generated, other qualifying conditions being equal. This important blockage-ratio concept will be further discussed in a subsequent paper along with numerical and experimental results.

As presented above, both the gcB and the $\mathrm{cKdV}$ models are written in terms of section-mean quantities. Since a typical wavelength is much greater than the characteristic length in the cross-flow plane, these section-mean equations provide a good representation of the dominant features of the wave field. In addition, the detailed three-dimensional wave features in the cross-flow plane can nevertheless be recovered to leading order once we have solved the gcB or the $\mathrm{cKdV}$ equations for the section-mean motion with given initial and boundary conditions, as has been demonstrated above by (96) and shown in figure 5 .

We have applied our models to study the propagation of long waves travelling in a variable channel to simulate ocean waves running up into shallow water with variable depth and width. The conservation properties and also the validity of the two models will be further examined when our models are applied to study these more general problems. The theoretical, numerical and experimental results for these problems will be presented in a subsequent paper.

We appreciate very much the stimulating discussions with Dr George T. Yates and other members of the Engineering Science group at Caltech. We are also indebted to Professor M. C. Shen for his comments and suggestions. This work was jointly supported by NSF Grant MSM-8706045 and its successor 4DMS-8901440 and by ONR Grant N00014-89-J-1971.

\section{REFERENCES}

Benjamin, T. B. \& Mahony, J. J. 1971 On an invariant property of water waves. J. Fluid Mech. 49, 385-389.

CAI, Y. H. \& Shen, M. C. 1985 The development of a solitary wave in a channel of variable elliptical cross section. Phys. Fluids 28, 2352-2356.

Chang, P., Melville, W. K. \& Miles, J. W. 1979 On the evolution of a solitary wave in a gradually varying channel. J. Fluid Mech. 95, 401-414.

Daily, J. W. \& Stephan, S. C. 1952 The solitary wave. In Proc. Third Conf. on Coastal Engineering, pp. 13-30.

David, D., Levi, D. \& Wivternitz, P. 1987 Integrable nonlinear equations for water waves in straits of varying depth and width. Stud. Appl. Maths 76, 133-168.

Fenton, J. D. 1973 Cnoidal waves and bores in uniform channels of arbitrary cross-section. J. Fluid Mech. 58, 417-434.

Johnson, R. S. 1973 On the development of a solitary wave moving over an uneven bottom. Proc. Camb. Phil. Soc. 73, 183-203.

KeUlegan, G. H. 1948 Gradual damping of solitary waves. J. Res. Natl Bur. Stand. 40, 457-487.

Keulegan, G. H. \& Patterson, G. W. 1940 Mathematical theory of irrotational translation waves. J. Res. Natl Bur. Stand. 24, 47-101, PR1272.

LEE, S. J., YATES, G. T. \& WU, T. Y. 1989 Experiments and analyses of upstream-advancing solitary waves generated by moving disturbances. J. Fluid Mech. 199, 569-593. 
Madsen, O. S. \& MEI, C. C. 1969 The transformation of a solitary wave over an uneven bottom. J. Fluid Mech. 39, 781-791.

Mathew, J. \& Akylas, T. R. 1990 On three-dimensional long water waves in a channel with sloping sidewalls. $J$. Fluid Mech. 215, 289-307.

MiLes, J. W. 1976 Damping of weakly nonlinear shallow-water waves. J. Fluid Mech. 76, 251-257.

MILES, J. W. 1979 On the Korteweg-de Vries equation for a gradually varying channel. J. Fluid Mech. 91, 181-190.

MiLes, J. W. 1980 Solitary waves. Ann. Rev. Fluid Mech. 12, 11-43.

Pederson, G. \& GJevik, B. 1983 Run-up of solitary waves. J. Fluid Mech. 135, 283-299.

Peregrine, D. H. 1967 Long waves on a beach. J. Fluid Mech. 27, 815-827.

Peregrine, D. H. 1968 Long waves in a uniform channel of arbitrary cross-section. J. Fluid Mech. $32,353-365$.

Peters, A. S. 1966 Rotational and irrotational solitary waves in a channel with arbitrary cross section. Commun. Pure Appl. Maths 19, 445-471.

Shen, M. C. 1968 Long waves in a stratified fluid over a channel of arbitrary cross section. Phys. Fluids 11, 1853-1882.

ShEN, M. C. 1969 Asymptotic theory of unsteady three-dimensional waves in a channel of arbitrary cross section. SIAM J. Appl. Maths 17, 260-271.

Shen, M. C. \& Zhong, X. C. 1981 Derivation of $K-d V$ equations for water waves in a channel with variable cross section. J. Méc. 20, 789-801.

Shuto, N. 1974 Nonlinear long waves in a channel of variable section. Coastal Engng Japan 17, 1-12.

Tena, M. H. 1990 Forced emissions of nonlinear water waves in channels of arbitrary shape. Ph.D. thesis, California Institute of Technology, Pasadena, CA.

Teng, M. H. \& WU, T. Y. 1990 Generation and propagation of nonlinear water waves in a channel with variable cross section. In Engineering Science, Fluid Dynamics, pp. 87-108. World Scientific.

WU, T. Y. 1979 Tsunamis-Proc. National Science Foundation Workshop (May 7-9, 1979), pp. 110-149. Pasadena: Tetra Tech. Inc.

WU, T. Y. 1981 Long waves in ocean and coastal waters. J. Engng Mech. Div. ASCE 107, 501-522.

WU, T. Y. 1987 Generation of upstream-advancing solitons by moving disturbances. $J$. Fluid Mech. 184, 75-99.

ZELT, J.A. 1986 Tsunamis: the response of harbours with sloping boundaries to long wave excitation. Ph.D. thesis, California Institute of Technology, Pasadena, CA.

Zelt, J. A. \& RAIchlen, F. 1990 A Lagrangian model for wave-induced harbour oscillations. J. Fluid Mech. 213, 203-225. 